\title{
A case of gastric hamartomatous inverted polyp resected endoscopically
}

Authors

Institutions
Moyu Dohi', Yasuyuki Gen², Mika Yoshioka'

${ }^{1}$ Department of Internal Medicine, Yasaka Hospital, Kyoto, Japan

${ }^{2}$ Department of Gastroenterology and Hepatology, North Medical Center, Kyoto Prefectural University of Medicine, Kyoto, Japan submitted 17. July 2015 accepted after revision 21. February 2016

\section{Bibliography}

DOI http://dx.doi.org/ 10.1055/s-0042-105201 Published online: 19.5.2016 Endoscopy International Open 2016; 04: E608-E609

(c) Georg Thieme Verlag KG Stuttgart · New York E-ISSN 2196-9736

\section{Corresponding author} Moyu Dohi, MD

Department of Internal Medicine

Yasaka Hospital

3452-1 Mizotani Yasaka-cho

kyotango-shi

Kyoto 627-0111

Japan

Fax: +81-772-65-4136

m03019mk@jichi.ac.jp
We report the case of a 55-year-old woman with a tumor in the greater curvature of the upper gastric body. The tumor was incidentally found on an upper gastrointestinal X-ray series performed during a routine medical examination. Whereas endoscopy revealed a gastric submucosal tumor (SMT), endoscopic ultrasonography demonstrated a heterogeneous tumor with small, cystic, hypoechoic spots originating from the second layer. The patient was clinically asymptomatic, with no contributory family history or abnormal labora-

\section{Introduction \\ $\nabla$}

Gastric hamartomatous inverted polyp (GHIP) is a rare form of gastric polyp. The accurate diagnosis of GHIP is difficult preoperatively because the main lesion is located in the submucosa or within the muscularis mucosae. Here, we report the successful resection of a submucosal tumor (SMT) type GHIP by endoscopic submucosal dissection (ESD).

\section{Case report}

A 55-year-old woman presented after a gastric tumor in the greater curvature of the upper gastric body had been detected incidentally on an upper gastrointestinal X-ray series performed during a routine medical examination. Endoscopy revealed a gastric SMT measuring $15 \mathrm{~mm}$ in diameter in the greater curvature of the upper gastric body. The elevated lesion was covered with normal mucosa, and an irregular depression was evident at the top of the SMT ( $\bullet$ Fig. 1). Endoscopic ultrasonography $(20 \mathrm{MHz})$ showed a heterogeneous tumor with small, cystic, anechoic spots originating from the second layer, indicating that the main lesion was located in the deep mucosal layer or within the muscularis mucosae ( $\bullet$ Fig. 2). A biopsy specimen from the depressed tory data. The results of a physical examination, abdominal computed tomography, and plain chest radiography were all unremarkable. Although the endoscopic tumor type was determined to be SMT, the tumor was successfully resected by endoscopic submucosal dissection (ESD) and subsequently diagnosed as a gastric hamartomatous inverted polyp (GHIP). The findings of the present case highlight the importance of considering GHIP as a diagnosis and indicate the utility of en bloc resection of GHIP with ESD.

area showed no neoplastic changes. The patient was clinically asymptomatic, with no contributory family history or abnormal laboratory data. Physical examination and abdominal computed tomography revealed no abnormalities.

To obtain an accurate diagnosis, the tumor was resected endoscopically. Because the tumor had the shape of an SMT, we considered that complete resection with conventional endoscopic mucosal resection (EMR) would be difficult. ESD was therefore selected to avoid piecemeal resection. We used an insulation-tipped diathermic knife (IT Knife2; Olympus, Tokyo, Japan) to perform an en bloc resection of the SMT by ESD without any complications ( Fig.3). On histopathological examination, the tumor was characterized by cystic, dilated, hypertrophic pseudopyloric gland proliferation. Smooth-muscle fibers were located in the submucosal layer, with branching from the proliferation of smooth-muscle bundles. The surface of the polyp was covered by gastric mucosa without atypia. According to these histological findings, the pathological diagnosis was GHIP (๑ Fig.4).

\section{Discussion}

\section{$\nabla$}

GHIPs account for fewer than $1 \%$ of all gastric polyps [1]. GHIPs are usually found as a part of 


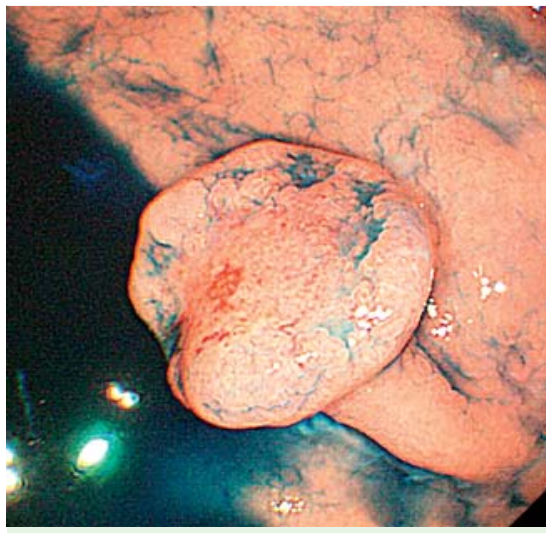

Fig. 1 Endoscopy shows a gastric submucosal tumor (SMT) measuring $15 \mathrm{~mm}$ in diameter in the greater curvature of the upper gastric body. An irregular depression and erosion are evident at the top of the SMT. The tumor was found incidentally in a 55-year-old woman on an upper gastrointestinal X-ray series performed during a routine medical examination.

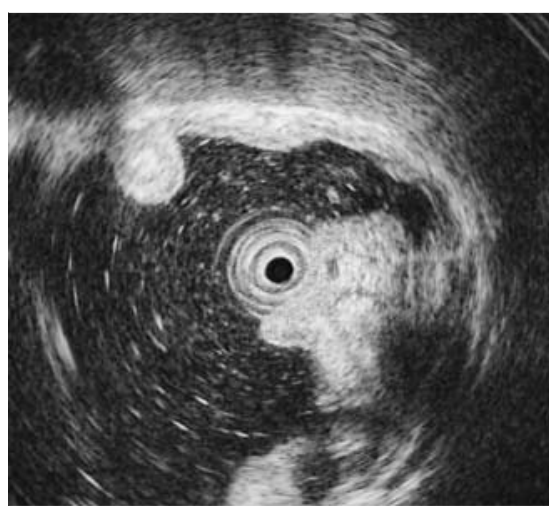

Fig. 2 Endoscopic ultrasonography shows a heterogeneous tumor with small, cystic, hypoechoic spots originating from the second layer.

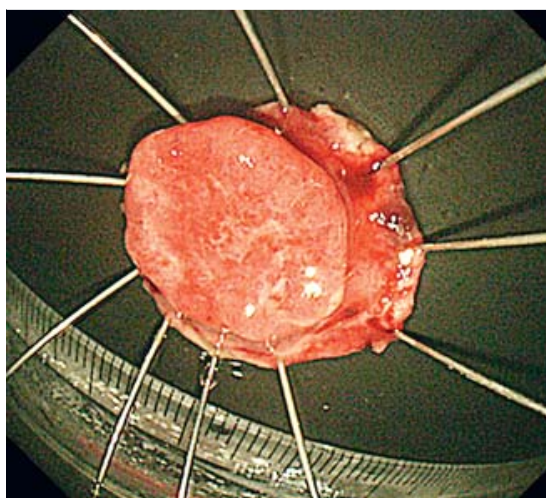

Fig. 3 The lesion is completely resected with a sufficient safety margin.

Peutz-Jeghers syndrome or Cowden disease but are rarely solitary or asymptomatic [2]. GHIPs are characterized pathologically by cystic, dilated, hypertrophic pseudopyloric gland proliferation and by smooth-muscle fibers located in the submucosal layer, with branching from the proliferation of smooth-muscle bundles. The main lesion in GHIP is located in the submucosa or within the muscularis mucosae [3].

GHIPs are difficult to diagnose accurately without endoscopic or surgical resection and subsequent pathological investigation because of the inverted growth into the submucosal layer [4]. GHIPs without a stalk are classified as of the SMT type, whereas those with a stalk are classified as of the polyp type. Polyp-type GHIPs can be resected endoscopically by conventional EMR, but conventional EMR has been reliable for resecting SMT-type lesions in one piece only if the lesions have a diameter of $10 \mathrm{~mm}$ or less. For SMT-type lesions larger than $10 \mathrm{~mm}$ in diameter, surgical resection is performed [5]. Furthermore, approximately $20 \%$ of GHIPs coexist with precancerous or cancerous areas [6-8]. In

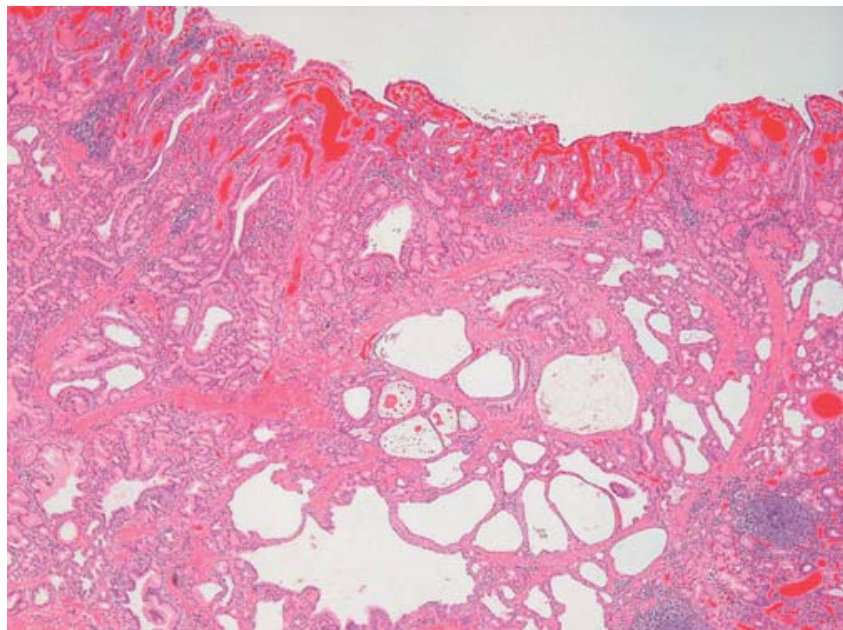

Fig. 4 Histopathological examination shows cystic, dilated, hypertrophic pseudopyloric gland proliferation and smooth-muscle fibers located in the submucosal layer, with branching from the proliferation of smooth-muscle bundles (hematoxylin and eosin, $\times 40$ ).

our patient, the diameter of the SMT was about $15 \mathrm{~mm}$, so we selected ESD rather than conventional EMR to achieve a complete en bloc resection and obtain an accurate pathological diagnosis. According to a search of the PubMed database, only four reports have described GHIP removal by ESD [4,7-9]. The resection of an SMT-type GHIP by ESD is thus quite rare. An accumulation of case reports for the endoscopic resection of GHIP is important, although the endoscopic and endoscopic ultrasound findings were relatively typical in the present case. ESD can provide sufficient tissue for an accurate pathological diagnosis following complete en bloc resection. We therefore think that for an accurate diagnosis of GHIP, en bloc resection with an ESD method is warranted.

\section{Competing interests: None}

\section{References}

1 Sipponen P, Siurara M. Cystic "hamartomatous" epithelial polyps of the stomach. Acta Hepatogastroenterol 1978; 25: 380-383

2 Jeghers H, McKusick VA, Katz KH. Generalized intestinal polyposis and melanin spots of the oral mucosa, lips and digits: a syndrome of diagnostic significance. N Engl J Med 1949; 241: 993

3 Itoh $K$, Tsuchigame T, Matsukawa T et al. Unusual gastric polyp showing submucosal proliferation of glands: case report and literature review. J Gastroenterol 1998; 33: 720-723

4 Hirohito $M$, Hideki K, Takaaki $T$ et al. Two rare gastric hamartomatous inverted polyp cases suggest the pathogenesis of growth. World J Gastroenterol 2014; 20: 5918-5923

5 Aoki M, Yoshida M, Saikawa Yet al. Diagnosis and treatment of a gastric hamartomatous inverted polyp: report of a case. Surg Today 2004; 34: $532-536$

$6 \mathrm{Oh} \mathrm{SJ}$, Oh CA, Kim DH et al. Adenocarcinoma derived from gastric hamartomatous polyps. J Korean Surg Soc 2011; 81: 419-422

7 Odashima $M$, Otaka $M$, Nanjo $H$ et al. Hamartomatous inverted polyp successfully treated by endoscopic submucosal dissection. Intern Med 2008; 47: 259-262

8 Ono S, Kamoshida T, Hiroshima Yet al. A case of early gastric cancer accompanied by a hamartomatous inverted polyp and successfully managed with endoscopic submucosal dissection. Endoscopy 2007; 39: E202

9 Matsuoka J, Itaba S, Makihara Y et al. Three cases of pedunculated gastric hamartomatous inverted polyps resected endoscopically. Nihon Shokakibyo Gakkai Zasshi 2015; 112: 1030-1036 